\title{
Identification of novel sense and antisense transcription at the TRPM2 locus in cancer
}

\author{
Ugo Orfanelli ${ }^{1}$, Ann-Kathrin Wenke ${ }^{2}$, Claudio Doglioni ${ }^{1}$, Vincenzo Russo ${ }^{1}$, Anja Katrin Bosserhoff ${ }^{2}$, Giovanni
} Lavorgna $^{1}$

${ }^{I}$ DIBIT - San Raffaele Scientific Institute, Via Olgettina 58, 20132 Milan, Italy; ${ }^{2}$ Institute of Pathology, University of Regensburg, Franz-Josef-Strauss-Allee 11, D-93053 Regensburg, Germany

It has been proposed that in cancer, where the bulk of the genome becomes hypomethylated, there is an increase in transcriptional noise that might lead to the generation of antisense transcripts that could affect the function of key oncosuppressor genes, ultimately leading to malignant transformation. Here, we describe the computational identification of a melanoma-enriched antisense transcript, TRPM2-AS, mapped within the locus of TRPM2, an ion channel capable of mediating susceptibility to cell death. Analysis of the TRPM2-AS genomic region indicated the presence in the same region of another tumor-enriched TRPM2 transcript, TRPM2-TE, located across a CpG island shared with TRPM2-AS. Quantitative PCR experiments confirmed that TRPM2-AS and TRPM2-TE transcripts were up-regulated in melanoma, and their activation was consistent with the methylation status of the shared CpG island. Functional knock-out of TRPM2-TE, as well as over-expression of wild-type TRPM2, increased melanoma susceptibility to apoptosis and necrosis. Finally, expression analysis in other cancer types indicated that TRPM2-AS and TRPM2TE over-expression might have an even wider role than anticipated, reinforcing the relevance of our computational approach in identifying new potential therapeutic targets.

Keywords: bioinformatics, transcription, apoptosis, antisense, methylation, ion channel

Cell Research (2008) 18:1128-1140. doi:10.1038/cr.2008.296; published online 28 October 2008

\section{Introduction}

There is overwhelming evidence indicating that human cancer is a genetic disease caused by sequential accumulation of mutations in oncogenes and tumor suppressor genes [1-3]. However, it is also increasingly apparent that cancer depends not only on genetic alterations, but also on epigenetic changes that collaborate with genetic traits to drive progressive stages of tumor evolution [4-6]. One of the main epigenetic cancer features is an altered DNA methylation pattern, composed of global demethylation and promoter-localized hypermethylation. While the latter phenomenon is more understood, a certain number of speculative hypotheses have been formulated about the former. For example, global hypomethylation of cancer

Correspondence: Giovanni Lavorgna

Tel: +39-02-26434776; Fax: +39-02-26434767

E-mail: giovanni.lavorgna@hsr.it

Received 22 February 2008; revised 29 April 2008; accepted 26 May 2008; published online 28 October 2008 cells could result in a de-repression of short interspersed repeated sequences (SINEs), long interspersed repeated sequences (LINEs) and retroviral sequences [7, 8]. Thereby, aberrant antisense RNA transcripts would be yielded, some of which could interfere with the function of key tumor suppressors [7], inhibiting their expression and promoting the evolution of malignant clones. Indeed, it would be interesting to systematically identify antisense transcripts whose expression is enriched in cancer cells, in order to study their possible role in carcinogenesis.

Over the past few years, we have developed a software program called AntiHunter, aimed at identifying antisense transcripts in the Expressed Sequence Tag (EST) database $[9,10]$. Recent algorithm improvements (Lavorgna et al., manuscript in preparation) have enabled us to apply AntiHunter to genome-wide identification of antisense transcripts in melanoma. Several antisense transcripts showing enrichment in melanoma were identified by the program (data not shown). Here, we report one of them, TRPM2-AS, mapped within the locus of TRPM2, an ion channel described as a member of the transient 
receptor potential (TRP) superfamily [11], which comprises a diverse group of voltage-independent calciumpermeable cation channels [12]. TRPM2 is activated by several second messengers [13-15], oxidative stress and TNF- $\alpha$ and is capable of mediating susceptibility to cell death [15-17, 18-20]. Moreover, a specific role for TRPM2 in cancer cell death has been recently proposed [21].

Intriguingly, graphical viewing of the AntiHunter results in a genomic browser indicated the presence of another tumor-enriched TRPM2 transcript in the same region, TRPM2-TE, located across a $\mathrm{CpG}$ island shared with TRPM2-AS. Expression analysis of TRPM2-AS and TRPM2-TE in malignant melanoma indicated that they were consistently up-regulated. It was also determined that TRPM2-TE could contribute to the down-regulation of TRPM2 function in cancer cells and that increasing the expression of wild-type TRPM2 in melanoma cells led to increased susceptibility to cell death. Finally, expression analysis in other cancer types indicated that TRPM2 silencing in cancer might have an even wider role than anticipated, reinforcing the relevance of our computational approach in identifying new molecules involved in the process of carcinogenesis.

\section{Results}

In silico identification of novel sense-antisense transcription at the TRPM2 locus

Using a newly developed version of our AntiHunter software, we performed a genome-wide search for antisense transcripts expressed in human ESTs from melanoma. Several antisense transcripts scattered throughout the genome were identified (data not shown) and here we describe one of them, named TRPM2-AS, mapped on chromosome 21. TRPM2-AS is antisense with respect to TRPM2, an ion channel capable of conferring cell death upon oxidative stress. AntiHunter identified two melanoma antisense ESTs, BF689755 and BF690298, located in the body of the TRPM2 gene and both corresponding to TRPM2-AS. The TRPM2 locus and the relevant molecules herein described are schematically represented in Figure 1 (numbering refers to the NCBI Build 36.1 (March 2006)). In order to confirm that the transcription start site and the 3 '-end of the ESTs were accurate, we performed 5'- and 3'-rapid amplification of cDNA ends (RACE) using a commercially available cDNA from malignant melanoma. As a result, it was possible to extend the $5^{\prime}$ end of TRPM2-AS by 292 bases, whereas the $3^{\prime}$ end matched closely with that of EST BF689755. The genomic span of the extended 875 nucleotides transcript (Genbank accession number: EU362988) was 10974 bp, ranging from position 44658901 to position 44669 874. A 701-bp CpG island, partially superimposed to the TRPM2-AS transcript, was mapped at coordinates 44 669 421-44 670121 by the "CpGplot" program from the EMBOSS package (www.emboss.org). A prediction for a 570-bp DNA segment containing a PolII promoter was made within the $\mathrm{CpG}$ island (coordinates 44669 48444670053 ) by "First-EF", a first-exon and promoter prediction program for human DNA [22], suggesting the $\mathrm{CpG}$ island could be involved in the transcriptional regulation of TRPM2-AS (Figure 1). Intriguingly, when visualizing the locus in a genomic browser, five ESTs from prostate carcinoma, BQ958319, BQ887469, BU543741, BM046691 and BQ920435, appeared to be possibly transcribed from the same CpG island/PolII promoter. (Only a representative one, $\mathrm{BC} 041570$, is shown for this group of ESTs in the upper part of Figure 1). Their transcription start site appeared to be located within intron 24 of the TRPM2 gene. In order to precisely map the $5^{\prime}$ - and the 3 '-ends of this transcript (named Tumor EnrichedTRPM2, herein TRPM2-TE), we performed another RACE experiment using cDNA from malignant melanoma. The 5'-end of TRPM2-TE was shown to expand by 22 bases with respect to EST BQ958319, whereas the 3 '-end was mapped in canonical position with respect to TRPM2 full-length transcript (herein TRPM2-FL). As a result, TRPM2-TE mRNA transcript was determined to be 2138 nucleotides long (Genbank accession number: EU362987), spanning a genomic region of 17553 nucleotides from position 44669840 to position 44687 392. Sequencing of the TRPM2-TE transcript revealed other interesting features. It lacked part of exon 26 and the entire exon 27. Removal of part of exon 26 does not affect the putative protein product, as the first methionine is positioned downstream (see below), whereas the ablation of exon 27 corresponds to the already described TRPM2- $\Delta \mathrm{C}$ splice variant [23]. The resulting deletion of 34 amino acids removes part of the Nudix domain and this has been reported to impair the capability of TRPM2-FL to be activated by ADP-ribose (ADPR, [23]). However, it leaves intact the segment showing similarity to the CORE region of the NUDT9 domain, containing all the structure required for ADPRase activity [24]. The predicted TRPM2-TE- $\Delta$ C open reading frame ranged from nucleotide 320 to nucleotide 874 , encoding for a 184 amino acid protein, whose predicted molecular size is $21083 \mathrm{Da}$. The full-length TRPM2-TE isoform without the $\Delta \mathrm{C}$ deletion encoded for a protein of 218 amino acids, with a predicted molecular mass of $25012 \mathrm{Da}$. A schematic representation of TRPM2-TE protein products, compared to TRPM2-FL, is shown in Figure 2. Interestingly, as shown in Figure 1, all five ESTs from prostate 


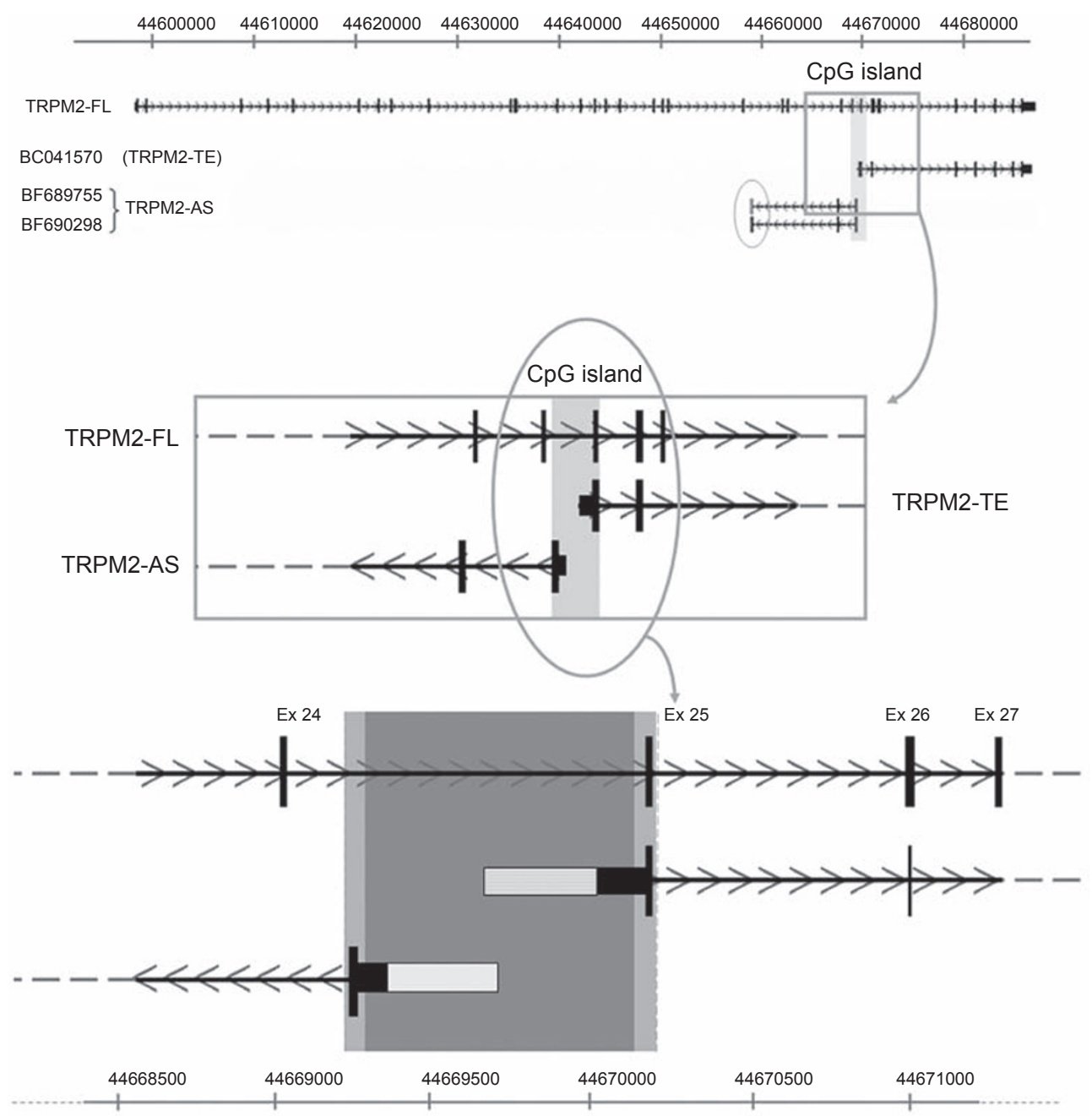

Figure 1 Identification of an antisense transcript and related molecules within the human TRPM2 gene on chromosome 21. (Top) A genome-wide search for melanoma antisense transcripts identified two antisense ESTs, BF689755 and BF690298 (circled), within the locus of TRPM2. The resulting antisense transcript mapped closely to an inner CpG island, predicted by the CpGplot program and shown in light gray, and nearby five sense ESTs from prostate carcinoma (only the representative EST BC041570 is shown), whose transcription seemed to originate on the other strand and in proximity to the antisense transcript. (Bottom) Primer extension experiments mapped the TRPM2-AS 5'-end in an overlapping position to the 5'-end of TRPM2-TE within intron 24 of full-length TRPM2 (TRPM2-FL). The primer-extended regions of TRPM2-AS and TRPM2-TE are shown as open bars. Sequence analysis of TRPM2-TE indicated the partial ablation of exon 26 and the removal of exon 27. The predicted $\mathrm{Pol}$ II promoter by the FirstExon program is shown in dark gray within the mapped CpG island. Sequences coordinates for TRPM2-AS and TRPM2-TE are: 44658 901-44 669874 and 44669 840-44 687 392, respectively. Numbering refers to the chromosomal position, whereas vertical bars indicate exons and arrows indicate the direction of transcription.

carcinoma displayed the same structural features as TRPM2-TE- $\Delta \mathrm{C}$, suggesting their possible association to cancer. However, selective amplification of the TRPM2TE- $\Delta$ C region from several melanoma samples revealed the co-existence of a mixed population of TRPM2-TE molecules, both with and without the $\Delta \mathrm{C}$ deletion in the same cell line (data not shown).

Expression analysis of TRPM2-AS, TRPM2-TE and

\section{TRPM2-FL transcripts in melanoma}

We analyzed TRPM2-AS and TRPM2-TE expression levels in 12 melanoma cell lines and five tumor tissues, with the cell line MaL being the immortalized counterpart of the fresh tumor \#3 (FT3). As a control, we used normal melanocytes from two independent healthy donors, NHEM1 and NHEM2. The expression level of these two transcripts was also measured in eight normal tissues: heart, brain, placenta, lung, liver, skeletal muscle, 

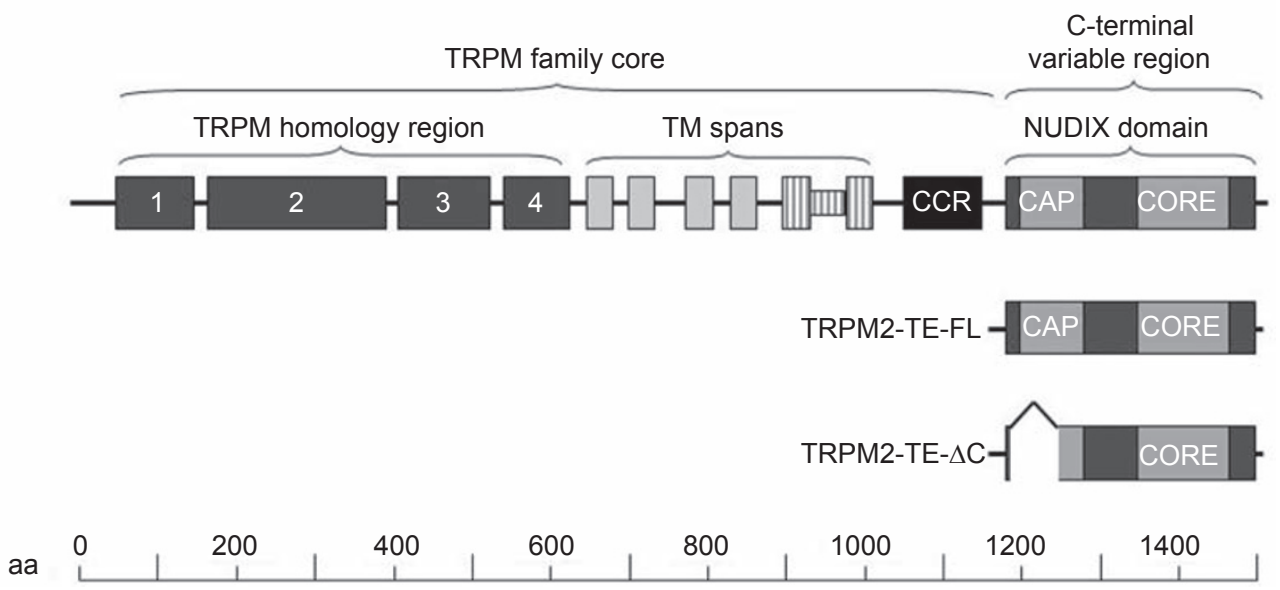

Figure 2 Schematic representation of the TRPM2-FL, TRPM2-TE-FL and TRPM2-TE- $\triangle \mathrm{C}$ protein products. The 'TRPM homology' domain and the 'TM' region indicate the region of highest similarity with other members of the TRPM family and the hydrophobic transmembrane span. The CORE and CAP regions within the NUDIX domain indicate the structure providing ADPRase activity and the enhancer of the CORE domain's affinity for ADPR, respectively. CCR: coiled coil region. The amino-acid scale is shown at the bottom of the figure.

kidney and pancreas. Results are shown in Figure 3A and $3 \mathrm{~B}$, respectively. Both molecules appeared to be up-regulated in over $80 \%$ of the cancer samples analyzed, with little or no detectable expression in normal melanocytes. The eight normal tissues analyzed displayed mostly low levels of these two transcripts. Interestingly, the level of up-regulation of both TRPM2-AS and TRPM2-TE molecules was similar in several of the tumor samples analyzed, suggesting a common mechanism of transcriptional regulation (see below). It should be noted that the MaL cell line displayed significantly lower levels of both TRPM2-AS and TRPM2-TE transcripts with respect to the corresponding fresh tumor sample, FT3. This indicates that quantification experiments performed in cell lines, especially after a high number of passages, might underestimate the level of up-regulation of these two molecules in original tumors. The expression level of TRPM2-FL was also measured in the same samples. Results are shown in Figure 3C. In this case, TRPM2-FL expression was detectable, albeit mostly at low levels, both in melanocytes and in 11 out of 12 melanoma cell lines. Strikingly, a much higher TRPM2-FL expression was instead observed in all tumor tissues. This discrepancy could be explained by the fact that fresh tumors are often infiltrated by immune cells, which are known to express fairly high levels of TRPM2-FL [13]. Alternatively, it is possible that immortalized cell lines have somehow been selected against expressing high levels of TRPM2FL, possibly owing to the proapoptotic nature of this gene [19-21].
Methylation status of the shared $C p G$ island correlates with the expression levels of TRPM2-AS and TRPM2-TE transcripts

We tried to ascertain if the degree of methylation of the $\mathrm{CpG}$ island shared by TRPM2-AS and TRPM2-TE was correlated with the transcriptional activation of these two molecules in melanoma. For this purpose, seven DNAs from the samples analyzed in Figure 3 were treated with sodium-bisulfite to convert cytosine residues to uracil in single-stranded DNA, under conditions whereby methylated cytosines remain essentially non-reactive. Primers were designed to PCR-amplify a region at coordinates 74-369 of the predicted $\mathrm{CpG}$ island. This region contains $19 \mathrm{CpG}$ dinucleotides, whose methylation status was determined after cloning and sequencing of at least 8 clones. The results are shown in Figure 4. Obvious differences in the methylation patterns of samples poorly expressing TRPM2-AS and TRPM2-TE, namely NHEM1 and NHEM2, vs relatively strong-expressing cell lines, i.e., Mel Juso, MR299, DettMel, MSR3 and Mel Im, were detected, suggesting that the methylation status of the $\mathrm{CpG}$ island contributes negatively to the expression level of both TRPM2-AS and TRPM2-TE.

Down-regulation of TRPM2-TE increases apoptosis and necrosis in melanoma

To analyze the functional role of TRPM2-TE in melanoma cells, we down-regulated its expression in melanoma cell line Mel Im by stable transfection with a TRPM2-TE antisense expression construct. Successful down-regulation of TRPM2-TE in cell clones 1 and 2 

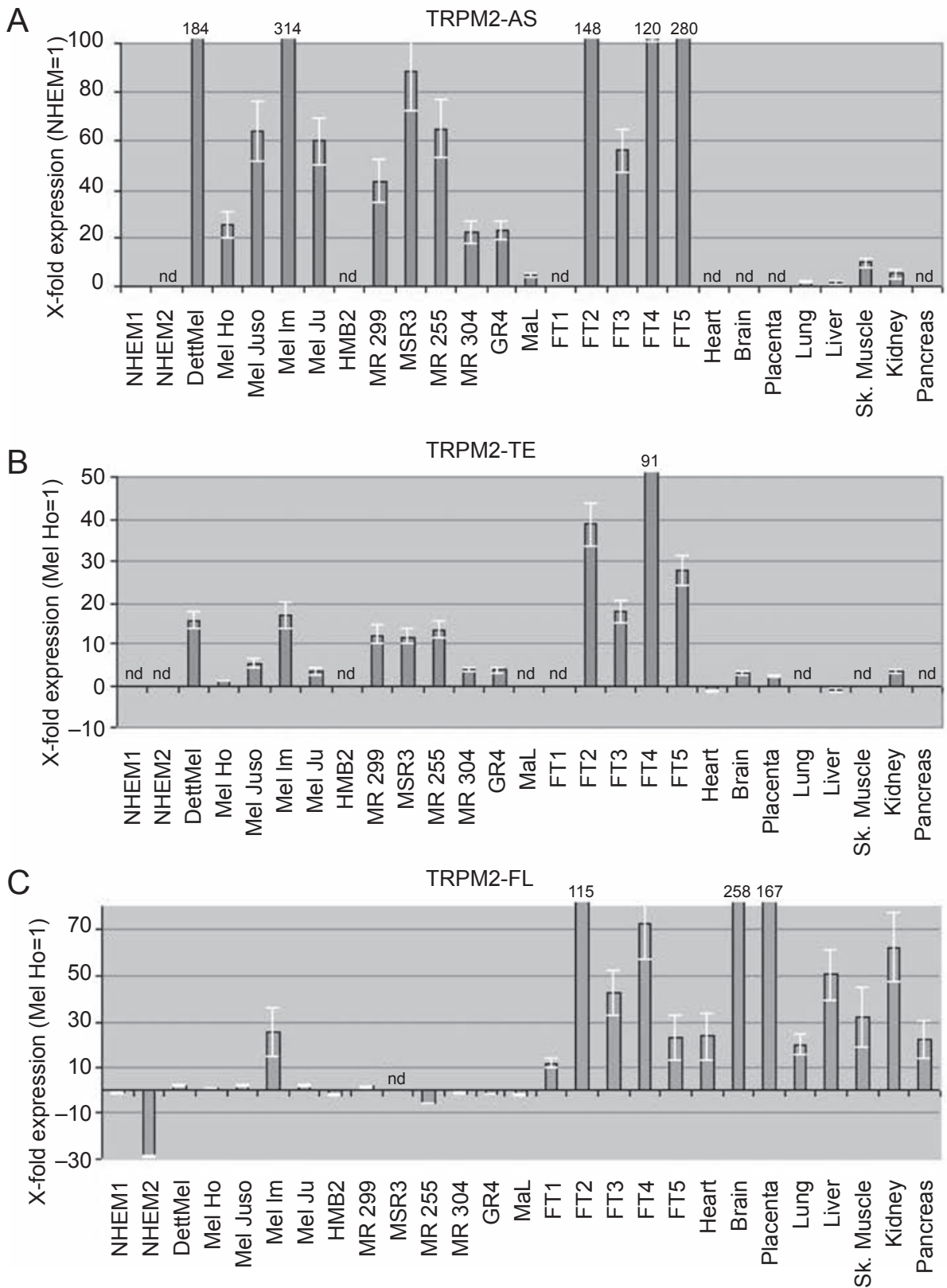

Figure 3 Expression analysis of TRPM2-AS, TRPM2-TE and TRPM2-FL transcripts in melanoma. Quantitative PCR experiments were performed to measure the relative amounts of TRPM2-AS (A), TRPM2-TE (B) and TRPM2-FL (C) transcripts in the following set of cDNA samples: two controls from normal human melanocytes, NHEM1 and NHEM2, two cell lines derived from a primary cutaneous melanoma, Mel Ho and Mel Juso, 10 cell lines derived from metastatic melanoma, HMB2, Mel Ju, Mel Im, DettMel, GR4, MaL, MR255, MR299, MR304, MSR3, five metastatic melanoma fresh tumors, FT1, FT2, FT3, FT4, FT5, eight normal tissues, heart, brain, placenta, lung, liver, skeletal muscle, kidney and pancreas. MaL cell line is the immortalized counterpart of the sample FT3. nd, not detected. Numbers on top of vertical bars indicate the actual off-scale value. Samples in (A) were normalized against NHEM1, whereas those in (B) and (C) were normalized against Mel Ho. The value of the normalizer sample was equated to 1 in all panels.

(TRPM2-TE-AS 1, TRPM2-TE-AS 2) was verified by quantitative RT-PCR (Figure 5A) and Western blot analysis (Figure 5B). As a side effect, the level of TRPM2FL was reduced by this treatment, as shown in Figure
5C. However, the down-regulation was certainly much more efficient for TRPM2-TE than for TRPM2-FL. We went on to analyze the extent of apoptosis in cell clones with down-regulated TRPM2-TE expression as well as in 


\begin{tabular}{|c|c|c|c|c|c|c|c|c|c|c|c|c|c|c|c|c|c|c|c|c|c|}
\hline & & 1 & & 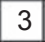 & 7 & 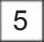 & 0 & 7 & 0 & 5 & 10 & 111 & 12 & 13 & 14 & 15 & 16 & 17 & 18 & 19 & \\
\hline & & & & & & & & & 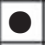 & & 0 & 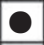 & 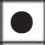 & 0 & 0 & 0 & 0 & 0 & 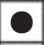 & 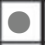 & \\
\hline 715 & & & & & & & & & & & 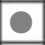 & & 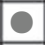 & 은 & 0 & 0 & 0 & 0 & 0 & & 0 \\
\hline I & & & & & 0 & 0 & 0 & 0 & 0 & 0 & 0 & 0 & 0 & 0 & 0 & 0 & 0 & 0 & 5 & & 27 \\
\hline R 299 & & 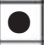 & 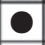 & 0 & 0 & 0 & 0 & 0 & 0 & 0 & 0 & 0 & 0 & 0 & 0 & 0 & 0 & 0 & 0 & 0 & .31 \\
\hline ettl & 84.22 & 0 & 0 & 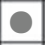 & 0 & 0 & 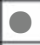 & 0 & 0 & 0 & 0 & 0 & 0 & 0 & 0 & 0 & 0 & 0 & ( & 0 & 5.66 \\
\hline B日 & & 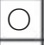 & 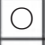 & & & 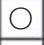 & & & & & 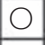 & 要 & 0 & 实 & 0 & - & 0 & - & 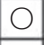 & - & 85 \\
\hline el In & .5 & 0 & 0 & 0 & 0 & 0 & 0 & 0 & 0 & 0 & 0 & 0 & 0 & 0 & 0 & 0 & 0 & 0 & 0 & 0 & 6.96 \\
\hline
\end{tabular}

Figure $4 \mathrm{CpG}$ methylation status of the inner TRPM2 CpG island in DNA from normal and tumor cells. DNAs from melanocytes (NHEM1 and NHEM2) and melanoma cell lines (Mel Juso, MR299, DettMel. MSR3, Mel Im) were treated with sodium bisulfite and subjected to sequencing of the TRPM2 inner CpG island. At least eight clones were sequenced for each sample. Open circles $(\bigcirc)$, gray circles $(\bigcirc)$ and closed circles $(O)$ indicate methylation in $0-29 \%, 30-79 \%$ and $80-100 \%$ of cases, respectively. The "AS fold" and "TE fold" columns represent the fold induction of TRPM2-AS and TRPM2-TE transcripts from Figure $3 \mathrm{~A}$ and $3 \mathrm{~B}$.

A

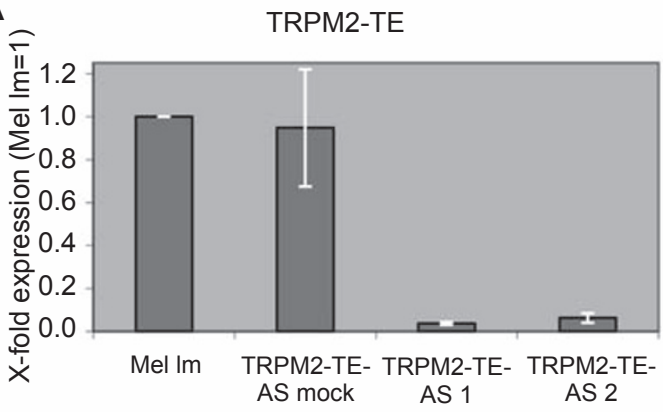

C

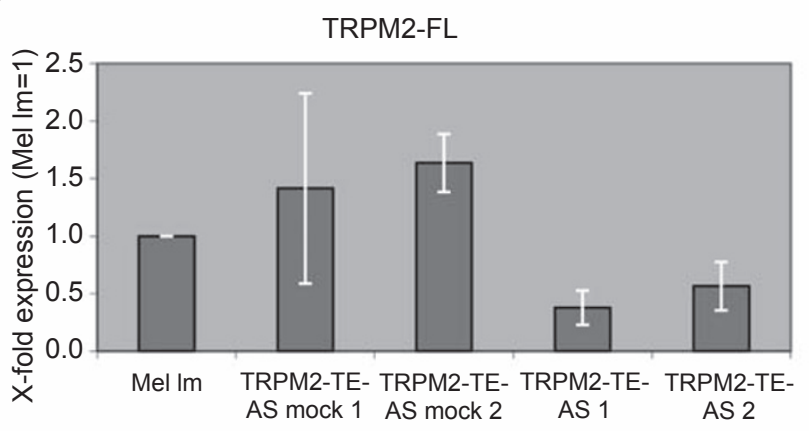

B

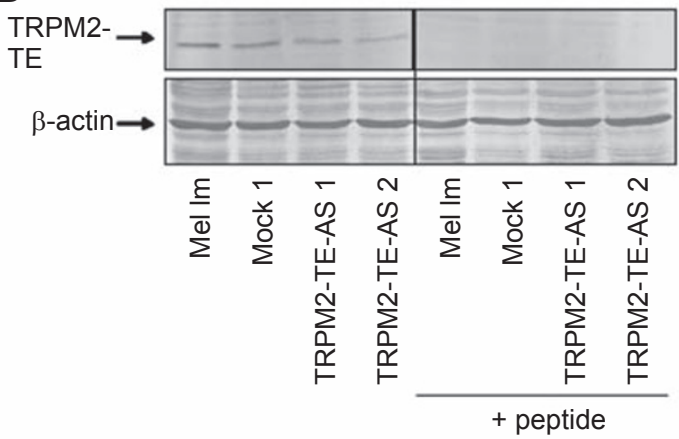

$\mathrm{D}$

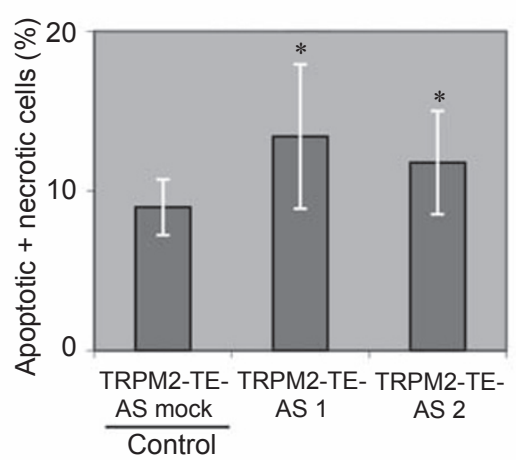

Figure 5 Down-regulation of TRPM2-TE expression in melanoma cell line Mel Im by stable transfection with a TRPM2-TE antisense expression construct. Successful down-regulation of TRPM2-TE in cell clones 1 and 2 (TRPM2-TE-AS 1, TRPM2TE-AS 2) was shown by quantitative RT-PCR (A) and western blot (B). In western blot analysis, the TRPM2-TE band was specifically competed by the peptide used for raising the antibody, but was not competed by an unrelated peptide (data not shown). $\beta$-Actin was used as a loading control. TRPM2-FL expression was partially reduced by the over-expression of the TRPM2-TE antisense construct. (C) The amount of apoptosis was measured in cell clones with down-regulated TRPM2-TE expression and in control cells. Without stimulation, TRPM2-TE-AS cell clones showed higher levels of apoptotic and necrotic cells compared to mock control cells. Stimulation with $\mathrm{H}_{2} \mathrm{O}_{2}$ enhanced this effect (data not shown) (D). $* P<0.05$ vs. control.

control cells. Without stimulation, TRPM2-TE-AS 1 and TRPM2-TE-AS 2 showed a higher level of apoptotic and necrotic cells compared to mock control cells, as shown in Figure 5D. Stimulation with $\mathrm{H}_{2} \mathrm{O}_{2}$ enhanced this effect (data not shown). 

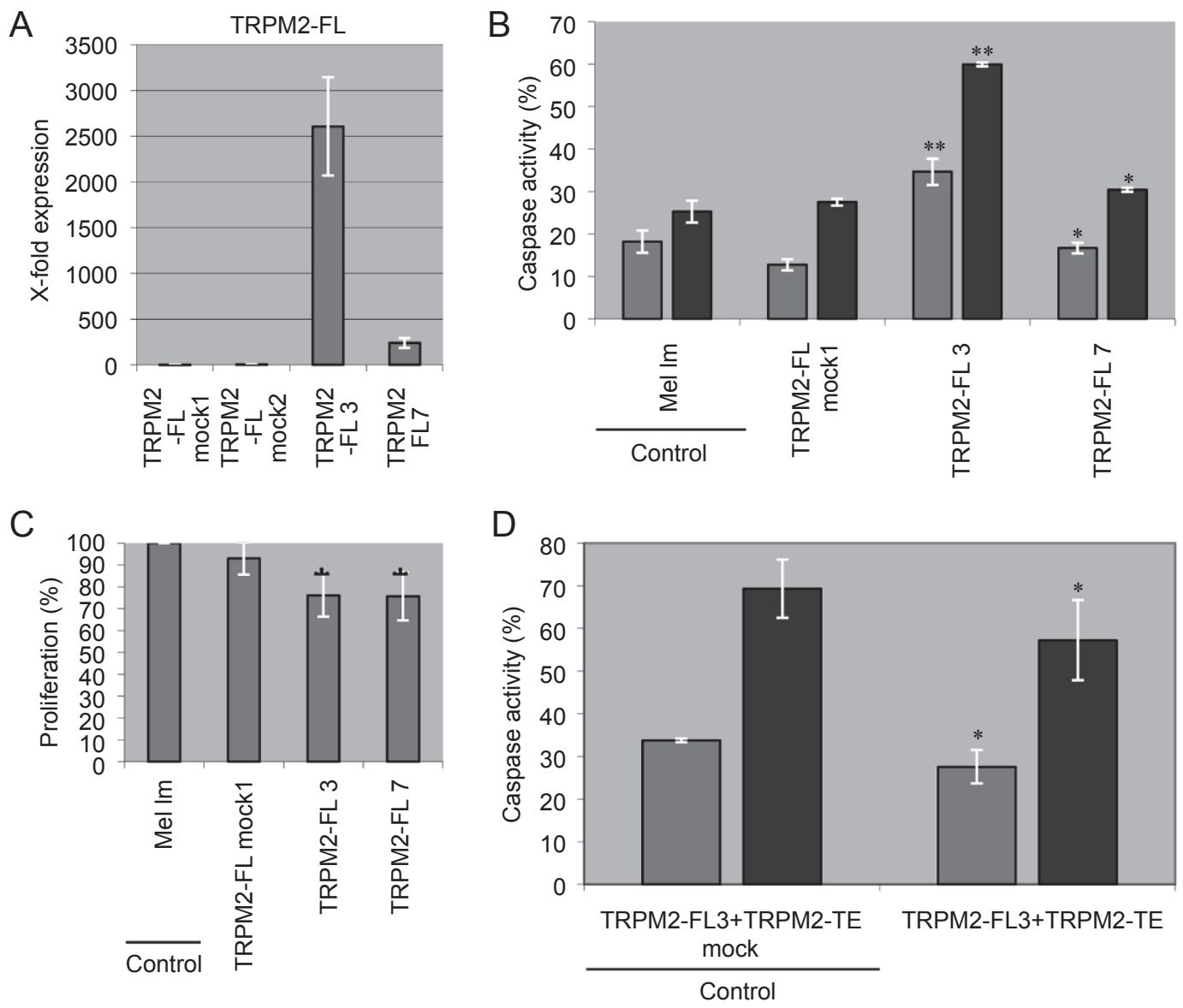

Figure 6 Analysis of melanoma cell clones with high expression of TRPM2-FL. Stable transfection of Mel Im cells with an expression construct for TRPM2-FL was confirmed by quantitative RT-PCR showing the successful over-expression of TRPM2FL in cell clones 3 and 7 compared to the mock-transfected cell clones mock 1 and mock 2 (A). TRPM2-FL cell clones, expressing high levels of TRPM2-FL, showed a longer doubling time in proliferation assays compared to mock-transfected cells and Mel Im cells (B). Measurement of apoptotic cells using a caspase assay showed that TRPM2-FL-expressing cell clones had higher levels of caspase activity compared to Mel Im cells and mock-transfected cell clones. This effect could be intensified by stimulating cells with $\mathrm{H}_{2} \mathrm{O}_{2}$ (C). Transient transfection of TRPM2-FL cell clone 3 with an expression plasmid for TRPM2-TE protected these cells from apoptosis. This effect was even stronger after stimulating cells with $\mathrm{H}_{2} \mathrm{O}_{2}$ (D). ${ }^{*} P<0.05$ vs. control; ${ }^{* *} P<0.01$ vs. control.

Over-expression of TRPM2-FL reduces melanoma proliferation and increases caspase activity

We generated melanoma cell clones which expressed higher levels of TRPM2-FL than parental cells by stable transfection of Mel Im cells with an expression construct for TRPM2-FL. Over-expression was tested by quantitative RT-PCR, revealing that the cell clones 3 and 7 had a strongly increased expression of TRPM2-FL when compared to mock-transfected cell clones mock 1 and mock 2 (Figure 6A). Cells expressing high levels of TRPM2FL showed reduced proliferation compared to mocktransfected cells and Mel Im cells (Figure 6B). These results are consistent with the analysis of the amount of apoptotic cells (data not shown). Strikingly, while clone
3 had greater expression than clone 7, there is no difference between these two clones in terms of proliferation rate. This is probably due to the fact that the capability to slow down cell proliferation via TRPM2-FL has been already saturated by the expression level reached using clone 7. Cell clones over-expressing TRPM2-FL had higher levels of caspase activity compared to Mel Im cells and mock-transfected cell clones. This effect could be intensified by stimulating cells with $\mathrm{H}_{2} \mathrm{O}_{2}$ (Figure 6C). Transient transfection of the TRPM2-FL cell clone 3 with a TRPM2-TE expression plasmid protected cells from apoptosis. This effect was even stronger after stimulating cells with $\mathrm{H}_{2} \mathrm{O}_{2}$, as shown in Figure 6D. 


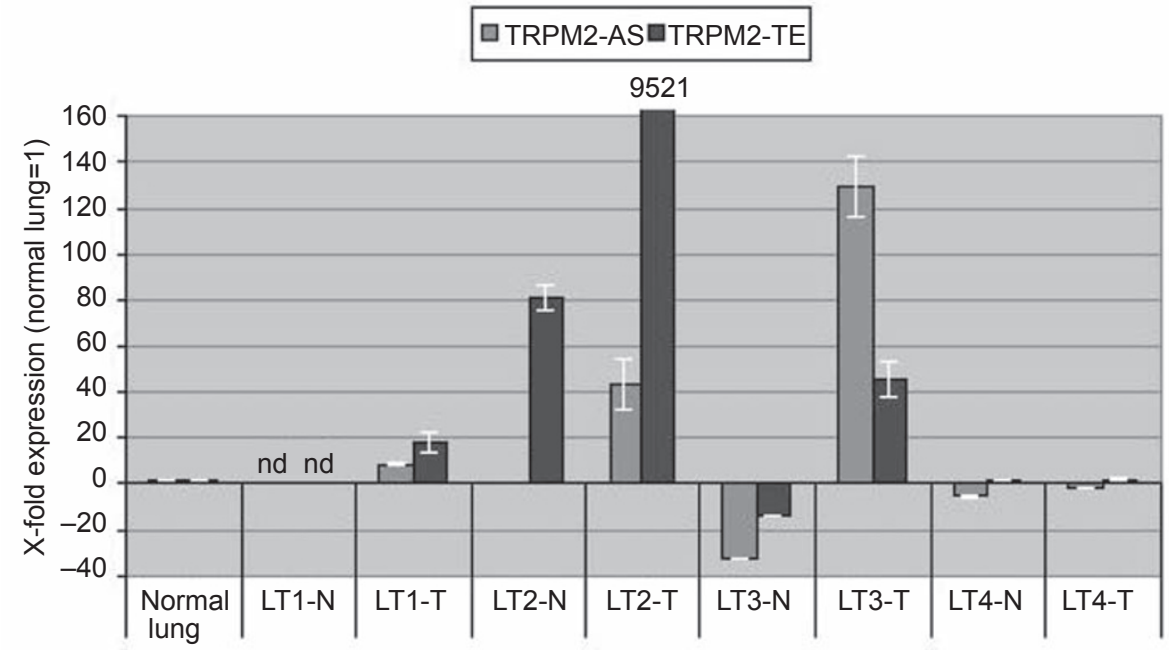

Figure 7 TRPM2-AS and TRPM2-TE are over-expressed in matched neoplastic/non-neoplastic samples of lung biopsies. Quantitative PCR experiments were performed to measure the relative amounts of TRPM2-AS (gray) and TRPM2-TE (dark) in four matched neoplastic non-neoplastic lung tissue (LT) samples, LT1-N and LT1-T, LT2-N and LT2-T, LT3-N and LT3-T, LT4-N and LT4-T. Results are shown as fold induction with respect to a lung cDNA from an independent healthy donor. nd, not detected.

TRPM2-AS and TRPM2-TE are also over-expressed in other tumor types

In order to investigate the possibility that overexpression of TRPM2-AS and TRPM2-TE transcripts is a wider phenomenon in cancer, quantitative RT-PCR experiments were performed on other tumor types. In Figure 7, four matched neoplastic/non-neoplastic lung tissue (LT) samples, with the normal tissue adjacent to the tumor, namely LT1-N and LT1-T, LT2-N and LT2-T, LT3-N and LT3-T, and LT4-N and LT4-T, were analyzed. Results are shown as fold induction with respect to a lung cDNA from an independent healthy donor. Tumor samples displayed higher levels of TRPM2-AS and TRPM2-TE transcripts with respect to their matched normal tissue. Interestingly, LT2 tissue sample, showing the highest TRPM2-TE levels among the cancer samples analyzed, also displayed high levels of this transcript in its healthy counterpart, suggesting the possibility that the spreading of tumor cells had already occurred in the ostensibly healthy tissue. Quantitative RT-PCR experiments were also performed to quantify TRPM2-AS and TRPM2-TE transcripts in both normal (HB100, MCF10) and breast cancer cell lines (MDAMB231, MDAMB453, MDAMB468, T47D, BT20, ZR751, MDA435, BT474, MCF7). Results are displayed as arbitrary units in Figure 8A and B, respectively. Quantification of TRPM2-AS revealed that it was undetectable in the two normal controls, as well as in MDAMB231, MDAMB453, ZR751 and MCF7 breast cancer cell lines. However, its expression was clearly up-regulated in the remaining five breast cancer cell lines (55\%). Expression analysis of TRPM2TE transcript showed that it was undetectable in the normal MCF10 cell line as well as in the MDAMB231 breast cancer cell line. It had a moderate expression in both normal HB100 and low-invasive MDAMB468 cell lines [25] and was significantly up-regulated in all of the remaining 7 cell lines (77\%).

\section{Discussion}

In the study presented here, we mined the melanoma EST database with our recently developed software AntiHunter and identified a new antisense transcript, TRPM2-AS. Natural antisense transcripts (NATs) are supposed to negatively regulate the corresponding sense transcript by means of a variety of regulatory mechanisms [9]. The sense partner of TRPM2-AS is TRPM2, a gene whose protein product encodes for an ion channel capable of conferring cell death upon oxidative stress. Although the direct involvement of TRPM2 in tumor cell death has been shown only very recently [21], our starting hypothesis already contemplated that activation of TRPM2-AS could interfere with the proapoptotic role of TRPM2 during the process of carcinogenesis.

Quantitative RT-PCR experiments revealed transcriptional activation of TRPM2-AS in about $80 \%$ of melanoma cell lines and tumor tissues vs. controls. Visualization of the AntiHunter output, using a genome browser, allowed us to hint at other interesting features of the investigated region. First, a $\mathrm{CpG}$ island/Pol II promoter, 
mapped within intron 24 of TRPM2, appeared to be closely positioned to the TRPM2-AS transcription start site. Second, five ESTs from prostate carcinoma appeared to have a transcription start site located within the same intron 24, suggesting the $\mathrm{CpG}$ island could serve also as a promoter for a new tumor enriched TRPM2 transcript (TRPM2-TE). Quantitative RT-PCR experiments showed that TRPM2-TE expression was activated in melanoma and coupled to the expression of TRPM2-AS, suggesting a bidirectional role for the shared promoter region. Sequencing of bisulfite-treated DNAs of several melanoma samples indicated the methylation status of the $\mathrm{CpG}$ island was at least partially correlated with the expression levels of TRPM2-AS and TRPM2-TE transcripts. Since this $\mathrm{CpG}$ island appeared to be mostly hypermethylated in normal melanocytes, it is conceivable that its demethylation could activate TRPM2-AS and TRPM2-TE expression in melanoma, which might have contributed to the functional down-regulation of TRPM2-FL.

No obvious indication on the regulatory role of TRPM2-AS over TRPM2-FL could be determined by the qPCR data shown in Figure 3A and 3C. However, as TRPM2-FL expression depends also on the strength of its own promoter, the effect of TRPM2-AS on TRPM2-FL expression may not be easily detected. Therefore, more compelling evidence must await experimental knock-out of TRPM2-AS in melanoma cells. Several mechanisms involved in the regulation of a sense transcript by its antisense partner have already been described (reviewed in [9, 26-30]): (1) transcriptional interference; (2) RNA masking; (3) double-stranded RNA (dsRNA)-dependent mechanisms and (4) antisense RNA-mediated CpG island methylation. Intriguingly, a recent paper reported the epigenetic silencing of the tumor suppressor gene $\mathrm{p} 15$ by its antisense RNA through heterochromatin formation, but not DNA methylation [31]. Although our results do not allow definition of the precise mechanism of action of TRPM2-AS, some of the above mechanisms are likely to be ruled out. Direct sequencing of several TRPM2-FL cDNAs failed to detect any deamination (A to I conversion) in the region encompassing exons 21-24, i.e., those overlapped by the primary TRPM2-AS transcript (data not shown), which suggests that RNA editing is unlikely to occur. Since primer extension experiments mapped the TRPM2-AS 3 '-end in the body of the TRPM2-FL gene, about $60 \mathrm{~kb}$ apart from its 5 '-end, antisense RNAmediated $\mathrm{CpG}$ island methylation can be excluded.

While a mechanism of action has yet to be found for TRPM2-AS, we have presented experimental evidence for the dominant-negative role of TRPM2-TE. Knockdown of this transcript significantly increased the susceptibility of melanoma cells to apoptosis and necrosis.
Since our experimental approach of TRPM2-TE depletion also removed a significant fraction of TRPM2-FL from the cells (see Figure 5B), the dominant negative role of TRPM2-TE could have been underestimated by our experiments. Over-expression of TRPM2-FL in melanoma cell clones led to an increased doubling time in proliferation assays as well as higher caspase activity. Along the same lines, transient over-expression of TRPM2-TE in the TRPM2-FL over-expressing cell clones protected these cells from apoptosis, suggesting that restoration of TRPM2-FL activity in melanoma cells could contribute to shifting them toward a less aggressive state and/or apoptosis. The mechanism by which TRPM2-TE inhibits the function of TRPM2-FL has yet to be defined. However, other truncated isoforms of TRPM2-FL have already been shown to act in a domi-
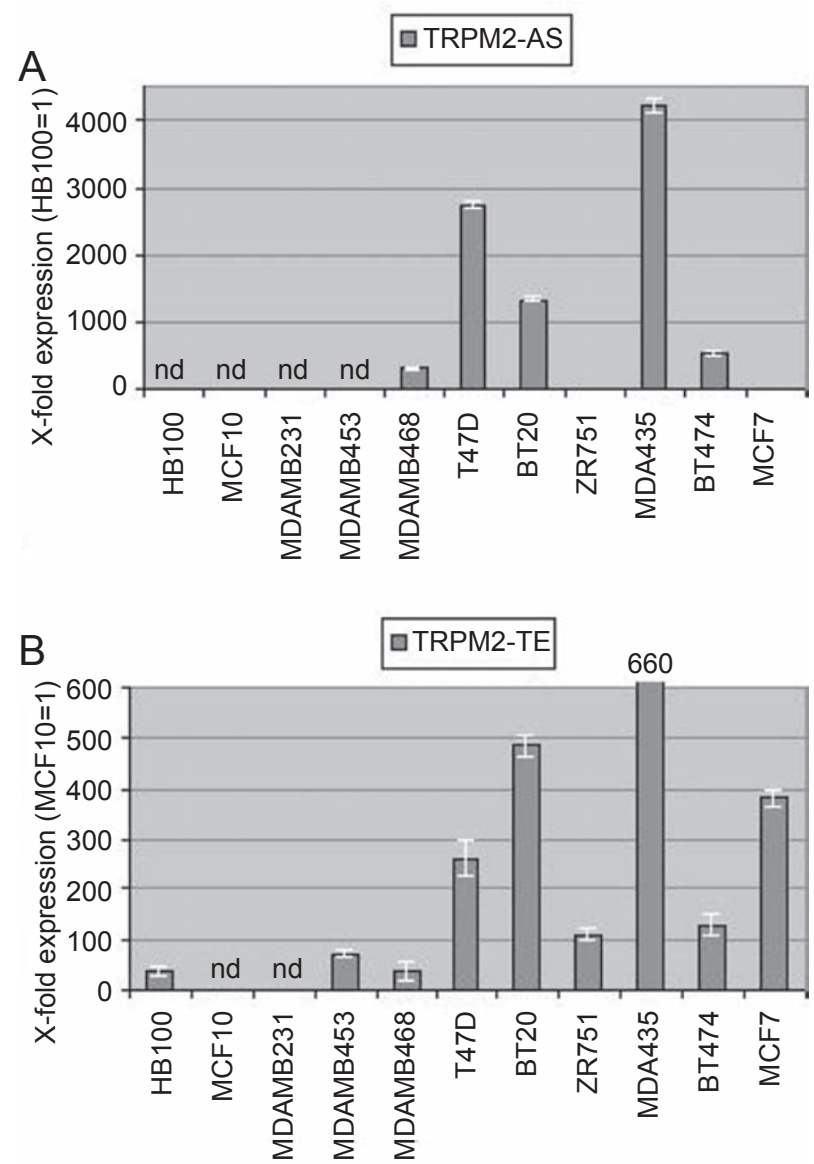

Figure 8 TRPM2-AS and TRPM2-TE are over-expressed in several breast cancer cell lines. Quantitative RT-PCR experiments were performed to determine the relative amount of TRPM2-AS (A) and TRPM2-TE (B) transcripts in normal (HB100, MCF10) and breast cancer cell lines (MDAMB231, MDAMB453, MDAMB468, T47D, BT20, ZR751, MDA435, BT474, MCF7). Results are shown as arbitrary units. nd, not detected. 
nant negative fashion $[17,32]$. Alternatively, or in adjunct, since TRPM2-TE retains the CORE portion of the Nudix domain required for ADPRase activity (see Figure 2 ), it might simply work by sequestering/consuming the cellular supply of ADPR needed for TRPM2 activation. In this respect, it will also be important to evaluate whether TRPM2-TE-FL and TRPM2-TE- $\Delta \mathrm{C}$ function differently.

Quantitative RT-PCR experiments performed in lung and breast cancers (Figures 7 and 8 ) seem to support the idea that activation of TRPM2-AS and TRPM2-TE transcripts is a phenomenon common to other tumors. However, before drawing any general conclusion, large patient cohorts with a good stratification in terms of clinical parameters will need to be analyzed in several other cancer types.

A large sequencing effort directed toward human colorectal and breast cancer DNA gene coding regions and splicing consensuses failed to find any mutation within TRPM2-FL [2]. Therefore, it is conceivable that TRPM2-FL inactivation in cancer is mainly obtained by epigenetic means, such as those described in this paper. A possible explanation is that TRPM2-FL function could be needed in the initial phases of tumor development, while becoming harmful at later stages. Accordingly, since metastasis is a cellular heterogeneous process, it is unlikely to be mediated by permanent genetic mutations. However, epigenetically mediated gene silencing is an excellent candidate for supporting such cellular dynamics [33]. The kind of epigenetic regulation hypothesized in this paper would allow the fine tuning of TRPM2-FL function in tumor cells by acting on the methylation status of the inner $\mathrm{CpG}$ island.

From the data reported here, restoration of TRPM2-FL activity in cancer cells could be an attractive therapeutic opportunity. Since inactivation of TRPM2-FL is likely to correlate with the hypomethylation of the $\mathrm{CpG}$ island shared by TRPM2-AS and TRPM2-TE transcripts, a simple way to restore its activity would be to artificially methylate this island de novo. The feasibility of this approach was shown by delivering a methylated oligonucleotide complementary to the targeted $\mathrm{CpG}$ island to the nucleus [34]. Preliminary attempts in our lab to deliver a methylated oligonucleotide to the shared $\mathrm{CpG}$ island have thus far been unsuccessful (data not shown), possibly owing to the high transcription occupancy of this region. As shown in Figure 1C, about half of the targeted $\mathrm{CpG}$ island is occupied by transcription on one strand, whereas the other half is occupied by transcription on both strands. This probably hinders the access of the oligonucleotide to its target sequence. We have planned a new series of experiments aimed at using higher oligo- nucleotide concentrations and different target sequences.

Systematic identification of antisense transcripts in the EST database holds great promise for discovering new and interesting biological and pathological phenomena. With the advent of new sequencing technologies that generate order-of-magnitudes larger sequencing data at fractional costs [35], it is conceivable that identification of regulatory antisense transcripts by mining the EST database will gain even more ground in the future.

\section{Materials and Methods}

\section{Computational identification of antisense transcripts in melanoma}

A new version of AntiHunter software, capable of performing genome-wide searches for antisense transcripts (Lavorgna et al., manuscript in preparation), was used for the identification of antisense ESTs from melanoma. Antisense ESTs identified by the program were visualized in their genomic context using the UCSC genome browser (http://genome.ucsc.edu/), in order to prioritize for further analysis those showing expression enriched in cancer tissues.

\section{Cells and cell culture}

The melanoma cell lines Mel Ho, Mel Juso, Mel Ju, Mel Im and HMB2 were described previously [36], whereas the cell lines DettMel, GR4, MaL, MR255, MR299, MR304 and MSR3 were from Hospital San Raffaele melanoma patient's' repository. The cell lines Mel Ho and Mel Juso were derived from primary cutaneous melanomas; Mel Im, Mel Ju, HMB2, DettMel, GR4, MaL, MR255, MR299, MR304 and MSR3 were derived from metastases of malignant melanomas. Cells and normal human epidermal melanocytes (NHEM) were cultured as described previously [37].

\section{Isolation of tumorous and non-tumorous human tissues}

Tissue samples from primary human melanoma and melanoma metastasis obtained from patients undergoing surgical treatment were immediately snap frozen and stored at $-80^{\circ} \mathrm{C}$. Informed consent was obtained from all patients and investigations were conducted according to institutional guidelines and according to the Declaration of Helsinki principles.

\section{RNA analysis}

Confluent cells $\left(1-5 \times 10^{6}\right)$ were trypsinized, pelleted and RNA extracted with RNeasy Mini Kit (Qiagen) according to the manufacturer's instructions. On-column DNase digestion was performed with $80 \mu$ of DNaseI (Invitrogen) for $40 \mathrm{~min}$ at room temperature. The eluted RNAs were quantified with a Nanodrop, while their integrity was controlled on a $1 \%$ agarose gel. Subsequently, cDNAs were generated by a reverse-transcriptase reaction performed using the ThermoScript RT-PCR System (Invitrogen). Briefly, $1 \mu \mathrm{g}$ of total cellular RNA, $1 \mu \mathrm{l}$ of dNTPs $(10 \mathrm{mmol} / \mathrm{l})$ and $1 \mu 1$ of dN6-primer $(50 \mathrm{ng} / \mu \mathrm{l})$ were mixed and incubated for $5 \mathrm{~min}$ at $65^{\circ} \mathrm{C}$. Then, $1 \mu \mathrm{l}$ of Thermoscript reverse transcriptase, $4 \mu \mathrm{l}$ of $5 \times$ first strand buffer, $1 \mu \mathrm{l}$ of $0.1 \mathrm{~mol} / \mathrm{l}$ DTT, $1 \mu \mathrm{l}$ of RNaseOUT $(40 \mathrm{U} / \mu \mathrm{l})$ and sterile RNase-free water were added to a $20 \mu \mathrm{l}$ total reaction volume. In order to estimate the amount of genomic DNA contamination in our preparations, each RNA was used to produce 
a parallel RT-minus reaction where the RT-enzyme was substituted with water. Reactions were incubated for $10 \mathrm{~min}$ at $25{ }^{\circ} \mathrm{C}$, and the RNAs were then transcribed for $1 \mathrm{~h}$ at $55^{\circ} \mathrm{C}$. Subsequently, reverse transcriptase was inactivated at $85^{\circ} \mathrm{C}$ for $5 \mathrm{~min}$ and RNA was degraded by digestion with $1 \mu \mathrm{l}$ of RNase $\mathrm{H}(2 \mathrm{U} / \mu \mathrm{l})$ at $37^{\circ} \mathrm{C}$ for $20 \mathrm{~min}$. cDNAs were controlled by PCR amplification of $\beta$ actin.

\section{5'- and 3'-RACE}

Human XG Malignant Melanoma (A375) marathon-ready cDNA (Clontech, Mountain View, CA) was used as template for PCR according to the manufacturer's instructions. Primer sequences are available on request. The largest-size PCR products were gel purified by the Illustra ${ }^{\mathrm{TM}}$ DNA and gel band purification kit (GE Healthcare, Buckinghamshire, UK) and cloned into a pGEM $^{\circledR}-\mathrm{T}$ Easy Vector System (Promega). Individual clones were sequenced using the DYEnamic ET Dye Terminator kit (Amersham Biosciences).

\section{Western blot analysis}

Aliquots of $3 \times 10^{6}$ cells were lysed in $200 \mu \mathrm{l}$ RIPA buffer (Roche) and incubated for $15 \mathrm{~min}$ at $4{ }^{\circ} \mathrm{C}$. Insoluble fragments were removed by centrifugation at $13000 \mathrm{rpm}$ for $10 \mathrm{~min}$ and the supernatant lysate was stored at $-20{ }^{\circ} \mathrm{C}$. For western blotting, $40 \mu \mathrm{g}$ protein lysates were loaded and separated on $12.75 \%$ SDS-PAGE gels and subsequently blotted onto a PVDF membrane (Biorad, Hercules, CA, USA). After blocking for $1 \mathrm{~h}$ with $3 \%$ BSA / TBST $\left(0.05 \%\right.$ Tween) the membrane was incubated for $16 \mathrm{~h}$ at $4{ }^{\circ} \mathrm{C}$ with the primary antibodies [polyclonal anti-TRPM2 antibody generated by Biogenes (Berlin, Germany), 1:2 000] and anti- $\beta$-actin (Sigma, Deisenhofen, Germany, 1:2 500)). The peptide sequence recognized by the TRPM2 antibody reads as follows: MEVYKGYMDDPRNT. Subsequently, the membrane was washed three times in TBST, incubated for $1 \mathrm{~h}$ with alkaline phosphate-coupled secondary antibody (Chemicon; 1:2 000) and then washed again. Finally, immunoreactions were visualized by NBT/BCIP (Sigma) staining.

\section{DNA constructs, transfections of target cells and functional assays}

A panel of Mel Im cell clones with reduced TRPM2-TE expression were established by stable transfection with an antisense expression plasmid (base -312 to +18 relative to the ATG codon cloned in antisense orientation into pCMX-PL1). Mel Im cell clones with induced TRPM2-FL expression were generated by stable transfection with a sense expression plasmid containing the coding sequence of TRPM2-FL from the translation start to the stop codon. Plasmids were cotransfected with pcDNA3 (Invitrogen), containing the selectable marker for neomycin resistance. Controls received pcDNA3 alone. Transfections were performed using Lipofectamin plus (Invitrogen) according to the manufacturer's instructions. One day after transfection, cells were placed in selection medium containing $50 \mu \mathrm{g} / \mathrm{ml} \mathrm{G} 418$ (Sigma). After 25 days of selection, individual G418-resistant colonies were subcloned. TRPM2-FL expression levels of these clones were checked in western blot and quantitative real-time PCR. Cell proliferation was determined using the XTT assay (Roche, Mannheim, Germany). Apoptotic cells were detected by staining with AnnexinV-FITS and propidium iodide using the AnnexinV
Kit (Caltag Laboratories/ Invitrogen, Karlsruhe, Germany) according to the manufacturer's manual; stained cells were measured by flow cytometry with the BD FACS Calibur System (BD Biosciences, San Jose, CA, USA). Data were analyzed and histograms generated using the Cellquest ${ }^{\mathrm{TM}}$ software (BD Biosciences). Measurement of caspase activity was another assay used to detect rates of apoptosis. Therefore cells were stained with CaspACE ${ }^{\mathrm{T}}$ ${ }^{\mathrm{M}}$ FITC-VAD-FMK In situ Marker (Promega, USA). In all, $5 \mu \mathrm{M}$ CaspACE ${ }^{\mathrm{TM}}$ FITC-VAD-FMK In situ Marker was added to the cell medium and cells were incubated for 20 min protected from light. After incubation, cells were washed with PBS twice and cell pellets were resuspended in $400 \mu \mathrm{l}$ PBS before analyzing by flow cytometry. To enhance apoptosis, $2 \times 10^{4}$ cells were seeded into each well of a six-well plate and stimulated with $2 \mathrm{mM} \mathrm{H}_{2} \mathrm{O}_{2}$ for $30 \mathrm{~min}$ before staining. To analyze the influence of the TRPM2-TE isoform on apoptosis, the TRPM2-FL-expressing cell clones were transiently transfected with an expression plasmid for TRPM2-TE. For transient transfections, $2 \times 10^{4}$ cells were seeded into each well of a six-well plate and transfected with $0.5 \mu \mathrm{g}$ TRPM2-TE plasmid DNA using the lipofectamine plus method (Invitrogen) according to the manufacturer's instructions. At $24 \mathrm{~h}$ after transfection, cells were stimulated with $2 \mathrm{mM} \mathrm{H}_{2} \mathrm{O}_{2}$ for 30 min and caspase activity was measured as described above. All experiments were performed at least three times.

\section{Analysis of DNA methylation}

Genomic DNA was isolated and purified with TRIzol reagent (Invitrogen) and quantified with a Nanodrop. On average $100 \mathrm{ng}$ of each DNA sample was subjected to bisulfite modification with the MethylEasy Kit (Human Genetic Signatures), allowing the sulfonation reaction to proceed for no less than $8 \mathrm{~h}$ at $55^{\circ} \mathrm{C}$. Following conversion, $3 \mu \mathrm{l}$ of the modified DNAs was PCR amplified with the primers $\mathrm{CpG}-8 \mathrm{U} 5^{\prime}$-TTG ATT TGA TTT GGT TTT TGG A-3' and CpG-8L 5'-CAA AAC AAA AAC TTC CTC TAT A-3' $(1 \mathrm{ng} / \mu \mathrm{l}$ each) in the presence of buffer 10× (Qiagen), dNTPs (2 mmol/1), $5 \times$ Solution Q (Qiagen) and Taq DNA Polymerase (3 U), with the reaction conditions being: $1 \mathrm{~min}$ of pre-incubation at $95^{\circ} \mathrm{C}$, then amplification reactions performed by 35 repetitive cycles of denaturing for $1 \mathrm{~min}$ at $95{ }^{\circ} \mathrm{C}$, annealing for $2 \mathrm{~min}$ at $55^{\circ} \mathrm{C}$ and extension for 2 min at $72{ }^{\circ} \mathrm{C}$. A semi-nested PCR reaction was performed in the same conditions except for the forward primer being changed to CpG-1U 5'-TGT AGA TTG TGT TTG AAT TTG GTT A-3', using $4 \mu 1$ of the previous 472 -bp PCR product as template. The resulting 370-bp product was then gel-purified, cloned and sequenced using standard procedures.

\section{Real-time quantification}

$\beta$-Actin (exons 5-6) was amplified from cDNAs using the specific primers: LC+hBactin-U 5'-TCC TCC TGG AGA AGA GCT A- $3^{\prime}$ and LC+hBactin-L $5^{\prime}$-GGA TGC CAC AGG ACT CCA T-3' coupled to human Universal Probe Library (UPL) probe number 11 (Roche), resulting in a 124-bp fragment. TRPM2-FL (exons 20-21) was amplified by RT-PCR from cDNAs using the specific primers: RCH_LONG_TRPM2_U 5'-ACC TCC TCA TCG CCA TGT T-3' and RCH_LONG_TRPM2 L 5'-CTT CCA AAT CTG GTC CGT GT-3' coupled to human UPL probe number 11, resulting in a 68-bp fragment. TRPM2-AS (exons 1-2) was amplified by RT-PCR from cDNAs using the specific primers: $\mathrm{RCH}$-asmel1F 5'-CCA GGA ACC AGA ACC AAA CT-3' and RCH-asmel- 
1R 5'-TGT CCG TCT GCT GAG ACA TC-3' coupled to human UPL probe number 57, resulting in a 61-bp fragment. TRPM2TE was amplified from cDNAs using the primers: UPL-TSS-U 5'-GAT GTT TTG GCG GAA GGA C-3' and UPL-TSS-Rev 5'CAG GAA GAC GTG ACG CAA G-3' coupled to human UPL probe number 6 , resulting in an 88 -bp intronless fragment. The contribution of genomic DNA contamination to the detected signal was subtracted by running a parallel real-time quantification from an RT-minus reaction, where the RT-enzyme was substituted with water. When RNA was not available to perform the RT-minus control, the contribution of DNA contamination to the detected signal was subtracted by performing a real-time quantification on the same samples, using a couple of primers specific for the $\beta$-actin promoter: LC-ProBACT-U1 5'-TCT GCA GGA GCG TAC AGA AC-3' and LC-ProBACT-L1 5'-ACA TCT CTT GGG CAC TGA GC-3' coupled to human UPL probe number 81 (Roche), resulting in a 85-bp fragment. Real-time PCR reactions were performed on a LightCycler 480 instrument (Roche), on 96 multiwell plates (Roche) using LightCycler 480 ProbeMaster Mix (Roche) following the manufacturer's instructions. Briefly, all reactions were performed in a final $30-\mu l$ volume containing $15 \mu \mathrm{l}$ of $2 \times$ MasterMix, $0.6 \mu \mathrm{l}$ of each primer $(20 \mu \mathrm{mol} / \mathrm{l}), 0.3 \mu \mathrm{l}$ of the corresponding UPL probe $(10 \mu \mathrm{mol} / \mathrm{l})$ and $10 \mu \mathrm{l}$ of template cDNA. Only for TRPM2AS the reaction was supplemented with $6 \mu$ of $5 \times$ Solution-Q (Qiagen) and the cDNA template lowered to $7 \mu \mathrm{l}$. The resulting mix was pre-incubated for $10 \mathrm{~min}$ at $95{ }^{\circ} \mathrm{C}$, then amplification reactions were performed by 45 repetitive cycles of denaturing for $10 \mathrm{~s}$ at $95{ }^{\circ} \mathrm{C}$, annealing for $15 \mathrm{~s}$ at $58{ }^{\circ} \mathrm{C}$ and a final extension plus single acquisition step at $72{ }^{\circ} \mathrm{C}$ for $1 \mathrm{~s}$. Levels of target gene transcripts were normalized to transcript levels of a reference gene $(\beta$-actin) and calculated using a relative quantification model with efficiency correction. Amplification efficiency of primer pairs was calculated from serial dilutions of a representative cDNA template over a concentration range of $3 \log$ orders.

\section{Statistical analysis}

Results are expressed as mean \pm SD (range) or percent. Comparison between groups was made using the Student's unpaired $t$-test. A $P$ value $<0.05$ was considered statistically significant. All calculations were performed using the GraphPad Prism software (GraphPad Software Inc, San Diego, USA). For real-time PCR, data analysis was performed using the relative expression software tool (REST 2005 BETA V1.9.12) [38] : ${ }^{*} P<0.05$; ** $P<0.01$.

\section{Acknowledgments}

We are grateful to Dr Francesca Sanvito (Fondazione Centro San Raffaele del Monte Tabor) for assistance with the pathological samples and to Sergio Bondanza (Istituto Dermopatico dell'Immacolata) for the generous gift of human melanocytes. The support of Prof Giorgio Casari (Fondazione Centro San Raffaele del Monte Tabor) is also gratefully acknowledged. This work is the subject of a US provisional patent application. GL was supported by the grants of Cariplo Foundation, MIUR and Alleanza Contro il Cancro, Italy.

\section{References}

1 Vogelstein B, Kinzler KW. Cancer genes and the pathways they control. Nat Med 2004; 10:789-799.

2 Sjoblom T, Jones S, Wood LD, et al. The consensus coding sequences of human breast and colorectal cancers. Science 2006; 314:268-274.

3 Wood LD, Parsons DW, Jones S, et al. The genomic landscapes of human breast and colorectal cancers. Science 2007; 318:1108-1113.

4 Baylin SB, Ohm JE. Epigenetic gene silencing in cancer - a mechanism for early oncogenic pathway addiction? Nat Rev Cancer 2006; 6:107-116.

5 Feinberg AP, Ohlsson R, Henikoff S. The epigenetic progenitor origin of human cancer. Nat Rev Genet 2006; 7:21-23.

6 Ting AH, McGarvey KM, Baylin SB. The cancer epigenome - components and functional correlates. Genes Dev 2006; 20:3215-3231.

7 Baylin SB, Herman JG. Alterations in cancer. In: Ehrlich M, ed. DNA. MA, USA: Eaton Publishing, 2000:293-309.

8 Ehrlich M. DNA hypomethylation and cancer. In: Ehrlich M, ed. DNA Alterations in Cancer. MA, USA: Eaton Publishing, 2000:273-291.

9 Lavorgna G, Dahary D, Lehner B, Sorek R, Sanderson CM, Casari G. In search of antisense. Trends Biochem Sci 2004; 29:88-94.

10 Lavorgna G, Triunfo R, Santoni F, Orfanelli U, Noci S, Casari G. AntiHunter 2.0: increased speed and sensitivity in searching BLAST output for EST antisense transcripts. Nucleic Acids Res 2005; 33:W665-W668.

11 Nagamine K, Kudoh J, Minoshima S, et al. Molecular cloning of a novel putative $\mathrm{Ca} 2+$ channel protein (TRPC7) highly expressed in brain. Genomics 1998; 54:124-131.

12 Clapham DE. TRP channels as cellular sensors. Nature 2003; 426:517-524.

13 Sano Y, Inamura $\mathrm{K}$, Miyake $\mathrm{A}$, et al. Immunocyte $\mathrm{Ca}^{2+}$ influx system mediated by LTRPC2. Science 2001; 293:1327-1330.

14 Kuhn FJ, Heiner I, Luckhoff A. TRPM2: a calcium influx pathway regulated by oxidative stress and the novel second messenger ADP-ribose. Pflugers Arch 2005; 451:212-219.

15 Perraud AL, Takanishi CL, Shen B, et al. Accumulation of free ADP-ribose from mitochondria mediates oxidative stressinduced gating of TRPM2 cation channels. J Biol Chem 2005; 280:6138-6148.

16 Hara Y, Wakamori M, Ishii M, et al. LTRPC2 $\mathrm{Ca}^{2+}$ permeable channel activated by changes in redox status confers susceptibility to cell death. Mol Cell 2002; 9:163-173.

17 Zhang W, Chu X, Tong Q, et al. A novel TRPM2 isoform inhibits calcium influx and susceptibility to cell death. $J$ Biol Chem 2003; 278:16222-16229.

18 Miller BA. The role of TRP channels in oxidative stressinduced cell death. J Membr Biol 2006; 209:31-41.

19 Yang KT, Chang WL, Yang PC, et al. Activation of the transient receptor potential M2 channel and poly(ADP-ribose) polymerase is involved in oxidative stress-induced cardiomyocyte death. Cell Death Differ 2006; 13:1815-1826.

20 Zhang W, Hirschler-Laszkiewicz I, Tong Q, et al. TRPM2 is an ion channel that modulates hematopoietic cell death through activation of caspases and PARP cleavage. Am J Physiol Cell 
Physiol 2006; 290:C1146-C1159.

21 Ishii M, Oyama A, Hagiwara T, et al. Facilitation of $\mathrm{H}_{2} \mathrm{O}_{2}$ induced A172 human glioblastoma cell death by insertion of oxidative stress-sensitive TRPM2 channels. Anticancer Res 2007; 27:3987-3992.

22 Davuluri RV, Grosse I, Zhang MQ. Computational identification of promoters and first exons in the human genome. Nat Genet 2001; 29:412-417.

23 Wehage E, Eisfeld J, Heiner I, Jungling E, Zitt C, Luckhoff A. Activation of the cation channel long transient receptor potential channel 2 (LTRPC2) by hydrogen peroxide. A splice variant reveals a mode of activation independent of ADP-ribose. $J$ Biol Chem 2002; 277:23150-23156.

24 Perraud AL, Shen B, Dunn CA, et al. NUDT9, a member of the Nudix hydrolase family, is an evolutionarily conserved mitochondrial ADP-ribose pyrophosphatase. J Biol Chem 2003; 278:1794-1801.

25 Thompson EW, Paik S, Brünner N, et al. Association of increased basement membrane invasiveness with absence of estrogen receptor and expression of vimentin in human breast cancer cell lines. J Cell Physiol 1992; 150:534-544.

26 Munroe SH. Diversity of antisense regulation in eukaryotes: multiple mechanisms, emerging patterns. $J$ Cell Biochem 2004; 93:664-671.

27 Werner A, Berdal A. Natural antisense transcripts: sound or silence? Physiol Genomics 2005; 23:125-131.

28 Lapidot M, Pilpel Y. Genome-wide natural antisense transcription: coupling its regulation to its different regulatory mechanisms. EMBO Rep 2006; 7:1216-1222.

29 Tufarelli C. The silence RNA keeps: cis mechanisms of RNA mediated epigenetic silencing in mammals. Philos Trans $R$ Soc
Lond B Biol Sci 2006; 361:67-79.

30 Mazo A, Hodgson JW, Petruk S, Sedkov Y, Brock HW. Transcriptional interference: an unexpected layer of complexity in gene regulation. J Cell Sci 2007; 120:2755-2761.

$31 \mathrm{Yu} \mathrm{W}$, Gius D, Onyango P, et al. Epigenetic silencing of tumour suppressor gene 15 by its antisense RNA. Nature 2008; 451:202-206.

32 Uemura T, Kudoh J, Noda S, Kanba S, Shimizu N. Characterization of human and mouse TRPM2 genes: identification of a novel N-terminal truncated protein specifically expressed in human striatum. Biochem Biophys Res Commun 2005; 328:1232-1243.

33 Jones PA, Baylin SB. The fundamental role of epigenetic events in cancer. Nat Rev Genet 2002; 3:415-428.

34 Yao X, Hu JF, Daniels M, et al. A methylated oligonucleotide inhibits IGF2 expression and enhances survival in a model of hepatocellular carcinoma. J Clin Invest 2003; 111:265-273.

35 Ryan D, Rahimi M, Lund J, Mehta R, Parviz BA. Toward nanoscale genome sequencing. Trends Biotechnol 2007; 25:385389.

36 Jacob K, Wach F, Holzapfel U, et al. In vitro modulation of human melanoma cell invasion and proliferation by all-transretinoic acid. Melanoma Res 1998; 8:211-219.

37 Poser I, Tatzel J, Kuphal S, Bosserhoff AK. Functional role of MIA in melanocytes and early development of melanoma. Oncogene 2004; 23:6115-6124.

38 Pfaffl MW, Horgan GW, Dempfle L. Relative expression software tool (REST) for group-wise comparison and statistical analysis of relative expression results in real-time PCR. Nucleic Acids Res 2002; 30:e36. 